\title{
On the combinatorics of suffix arrays
}

\author{
Gregory Kucherov $^{\mathrm{a}}$, Lilla Tóthmérész ${ }^{\mathrm{a}} \mathrm{b}, *$, Stéphane Vialette ${ }^{\mathrm{a}}$ \\ ${ }^{a}$ Université Paris-Est 8 CNRS, Laboratoire d'Informatique Gaspard Monge, Marne-la-Vallée, France \\ ${ }^{b}$ Loránd Eötvös University, Pázmány Péter sétány 1/C, H-1117 Budapest, Hungary
}

\begin{abstract}
We prove several combinatorial properties of suffix arrays, including a characterization of suffix arrays through a bijection with a certain well-defined class of permutations. Our approach is based on the characterization of Burrows-Wheeler arrays given in [1], that we apply by reducing suffix sorting to cyclic shift sorting through the use of an additional sentinel symbol. We show that the characterization of suffix arrays for a special case of binary alphabet given in 22] easily follows from our characterization. Based on our results, we also provide simple proofs for the enumeration results for suffix arrays, obtained in [3]. Our approach to characterizing suffix arrays is the first that exploits their relationship with Burrows-Wheeler permutations.
\end{abstract}

Keywords: combinatorics, permutations, suffix array, Burrows-Wheeler transform

\section{Introduction}

Suffix array is a very popular data structure in string algorithms, both in theoretical studies and practical applications, that has been designed as a space-efficient alternative to suffix trees [4, 5]. With the discovery of linear-time construction algorithms for suffix arrays [6, [7, 8], this data structure received an increasing attention during the last decade. A good deal of work has been devoted to improving the practical efficiency of suffix arrays.

A suffix array for a string of length $n$ is essentially a permutation of length $n$ corresponding to the starting positions of all suffixes sorted lexicographically. Obviously, if the alphabet has a fixed size $k<n$, then only a proper subset (at most $k^{n}$ ) of all $n$ ! permutations are suffix arrays for some word over this alphabet. A main motivation of this paper is to provide a characterization for suffix array permutations for bounded-size alphabets.

Our results take advantage of a very close relation between suffix arrays and the Burrows-Wheeler transform [9]. The Burrows-Wheeler transform of a string is a permutation of string letters which allows the string to be effectively reconstructed. Among other applications, it is the basis for many compact text indexes that have been intensively studied and used in practical applications (see e.g. [10] and references therein).

Crochemore et al. [1] pointed out a very nice characterization of Burrows-Wheeler arrays, which are close relatives of suffix arrays. This characterization, attributed to

${ }^{*}$ Corresponding author

Preprint submitted to Elsevier

November 20, 2018 
Gessel and Reutenauer [1], uses the key notion of linking permutation which is similar to (but different from) the notion of $\Psi$-function studied for suffix arrays [12]. We show that the approach of [1] can be successfully applied to obtain characterization results for suffix arrays, using a relation between orderings of suffixes and cyclic shifts.

As far as related works are concerned, He at al. 2] provided a characterization of suffix arrays for the case of binary alphabet $(k=2)$ and an assumption that the terminal sentinel symbol is ranked between the two main symbols in the alphabet ordering. We show that the characterization of [2] easily follows from the characterization that we propose in this paper.

In [3], Schürmann and Stoye prove several counting results for suffix arrays and corresponding strings. They use a characterization of suffix array permutations through $\Psi$-functions, that they call $R_{+}$-arrays, which are mappings from $[1, n]$ to $[0, n]$. A crucial parameter in countings is the number of descents in $R_{+}$-arrays, directly related to the minimal alphabet size on which the corresponding suffix array can be realized (see also [13]). Compared to our approach, the important difference is that the set of $R_{+}$-arrays is not characterized, while the set of linking permutations admit a neat combinatorial characterization as permutations with only one orbit. This allows us to provide a bijection between suffix arrays and a certain well-defined class of permutations. To demonstrate the usefulness of our approach, we obtain much simpler proofs of counting theorems from [3]. Our approach to characterizing suffix arrays is the first that exploits their relationship with Burrows-Wheeler permutations.

\section{Preliminaries}

In what follows, $\Sigma=\left\{a_{1}, a_{2}, \ldots, a_{k}\right\}$ is an ordered alphabet of size $k$, where $a_{1}<a_{2}<$ $\cdots<a_{k}$. The set of all permutations $\pi=\pi(1) \pi(2) \ldots \pi(n)$ of length $n$ is denoted by $\mathbf{S}_{n}$, and id denotes the identity permutation $12 \ldots n$. The composition of permutations $\sigma$ and $\pi$ is denoted $\pi \sigma$, i.e., $(\pi \sigma)(i)=\pi(\sigma(i))$. Throughout the paper, we assume that the addition (subtraction) of a constant value to a permutation value verifies the identities $n+1 \equiv 1,1-1 \equiv n$. In other words, $\pi(i)+k=((\pi(i)-1+k) \bmod n)+1$. For a permutation $\pi \in \mathbf{S}_{n}$, we let $\pi+k, k \in[1, n]$, stand for the permutation defined by $(\pi+k)(i)=\pi(i)+k$. Note that $\pi+k=(\mathrm{id}+k) \pi$. The permutation $(\pi-k)$ is defined similarly.

Definition 1 (suffix array). Given a word $w=w_{1} w_{2} \ldots w_{n}$ on alphabet $\Sigma$, its suffix array is a permutation $\pi$ such that $\pi(i)=j$ iff the suffix $w_{j} \ldots w_{n}$ is the $i^{\text {th }}$ in the lexicographic ordering of all suffixes of $w$.

For example, the suffix array of babba is 52413.

Definition 2 (primitive word). A word $u \in \Sigma^{+}$is called primitive if it is not a proper power of another word, i.e., $u=v^{n}, v \in \Sigma^{+}$, implies $n=1$.

Primitive words are exactly those words whose cyclic shifts are all distinct. Therefore, for a primitive word, we can consider the permutation defined by the lexicographic ordering of its cyclic shifts. We call this permutation the Burrows-Wheeler array because of its direct relation to the Burrows-Wheeler transform [9]. 
Definition 3 (BW-array). Given a primitive word $w=w_{1} \ldots w_{n}$, its Burrows-Wheeler array (hereafter $\mathrm{BW}$-array) is a permutation $\pi$ such that $\pi(i)=j$ iff the word $w_{j} \ldots w_{n} w_{1} \ldots w_{j-1}$ is the $i^{\text {th }}$ in the lexicographic ordering of all cyclic shifts of $w$.

For example, the BW-array of bbaba is 35241.

We write $\mathbf{S}_{n}^{c}$ for the set of all permutations of $\mathbf{S}_{n}$ with one orbit. The following notion has proved to be very helpful for characterizing $\mathrm{BW}$-arrays [11, 1]. It is related to $\Psi$-functions [12] or $R_{+}$-arrays [3] defined on suffix arrays, but defines a mapping on permutations.

Definition 4 (linking permutation, linking mapping). Let $\pi \in \mathbf{S}_{n}$. The linking permutation of $\pi$ is the permutation $\varphi=\pi^{-1}(\pi+1) \in \mathbf{S}_{n}^{c}$. The mapping $\pi \mapsto \varphi$ is called the linking mapping, and is denoted by $\Phi$.

As an example, the linking permutation of 52413 is 45123 . Observe that $\varphi \in \mathbf{S}_{n}^{c}$ follows from $\varphi\left(\pi^{-1}(i)\right)=\pi^{-1}(i+1)$, where $n+1 \equiv 1$. The linking permutation of a BWarray gives the ranks of the consecutive shifts in the lexicographic order. Furthermore, note that if $\pi(1)$ and $\varphi=\Phi(\pi)$ are known, then one can reconstruct $\pi$ by iterating $\pi(\varphi(i))=\pi(i)+1$ starting with $i=1$.

Definition 5. For two permutations $\pi$ and $\sigma$ of $\mathbf{S}_{n}$, define $\pi \sim \sigma$ if and only if there exists $k \in[1, n]$ such that $\sigma=\pi+k$.

It is easily seen that $\sim$ is an equivalence relation on $\mathbf{S}_{n}$.

Proposition 1. $\Phi$ is well-defined on $\mathbf{S}_{n} / \sim$ and bijective from $\mathbf{S}_{n} / \sim$ to $\mathbf{S}_{n}^{c}$.

Proof. We first show that $\Phi(\pi)=\Phi(\sigma)$ if $\pi \sim \sigma$. Let $\sigma=\pi+k$. Then, $\Phi(\pi)=$ $\pi^{-1}(\pi+1)=\pi^{-1}(((\pi+k)+1)-k)=\pi^{-1}(\mathrm{id}-k)((\pi+k)+1)$. Observe now that $(\pi+k)^{-1}=\pi^{-1}(\mathrm{id}-k)$. Therefore, $\Phi(\pi)=(\pi+k)^{-1}((\pi+k)+1)=\Phi(\pi+k)=\Phi(\sigma)$. This shows that $\Phi$ is well-defined on $\mathbf{S}_{n} / \sim$.

Second, we can uniquely determine $\pi$ from $\Phi(\pi)$ and $\pi(1)$, and hence $\Phi$ is injective on $\mathbf{S}_{n} / \sim$. The number of $\sim$-equivalence classes is $(n-1) !=\left|\mathbf{S}_{n}^{c}\right|$. Therefore, the mapping is a bijection.

Definition 6 (permutation descent). Let $\pi \in \mathbf{S}_{n}$. We say that $i \in[1, n-1]$ is a descent of $\pi$ if and only if $\pi(i)>\pi(i+1)$. The set of all descents of $\pi$ is denoted $\mathcal{D}(\pi)$.

The following theorem from [1] provides a nice characterization of BW-arrays through the linking mapping. It will play a central role in our study.

Theorem 1 ([1]). Let $r_{i} \geq 0,1 \leq i \leq k$, be integers such that $\sum_{i=1}^{k} r_{i}=n . \quad A$ permutation $\pi \in \mathbf{S}_{n}$ is the $B W$-array of a primitive word $w \in \Sigma^{n}$ with $r_{i}$ occurrences of letter $a_{i}, 1 \leq i \leq k$, if and only if $\mathcal{D}(\Phi(\pi)) \subseteq\left\{r_{1}, r_{1}+r_{2}, \ldots, r_{1}+\cdots+r_{k-1}\right\}$. Moreover, in this case $\pi$ is the $B W$-array of exactly one such word.

\section{Characterization of suffix arrays}

In this section, we state our characterization theorems for suffix arrays: Theorems 4, 5 and 6. We use a reduction of suffix sorting to cyclic shift sorting by appending a 
sentinel symbol to the end of the word, and thereby reduce the characterization of suffix arrays to the characterization of BW-arrays.

Consider a symbol $\sharp \notin \Sigma$, and the alphabet $\Sigma^{\prime}=\left\{\sharp, a_{1}, a_{2}, \ldots, a_{k}\right\}$ with $\sharp<a_{1}<$ $a_{2} \cdots<a_{k}$. We will examine the suffix arrays of words $w \sharp$ for $w \in \Sigma^{n}$. The following proposition is obvious.

Proposition 2. There is a one-to-one correspondence between the suffix arrays of $w \in$ $\Sigma^{n}$ and the suffix arrays of $w^{\prime} \in \Sigma^{n} \sharp$. If $\sigma \in \mathbf{S}_{n}$ is the suffix array of $w$, then $\pi \in \mathbf{S}_{n+1}$ is the suffix array of $w \sharp$ if and only if $\pi=(n+1) \sigma(1) \sigma(2) \ldots \sigma(n)$.

The following proposition shows, that for words in $\Sigma^{n} \sharp$, cyclic shift sorting is equivalent to suffix sorting. Note that this property remains true even if we do not assume that $\sharp$ is the smallest element in the ordering of $\Sigma \cup\{\sharp\}$. This will be important later.

Proposition 3. Let $w^{\prime}=w \sharp$, where $w \in \Sigma^{*}, \sharp \notin \Sigma$ and the ordering of $\Sigma \cup\{\sharp\}$ is arbitrary. Then $w^{\prime}$ is primitive, hence the order of its cyclic shifts is well-defined. Moreover the order of the cyclic shifts of $w \sharp$ coincides with the order of the suffixes of $w \sharp$.

Proof. If $w^{\prime}=u^{k}$ for some word $u$ and a $k>1$, then $k$ would divide the number of occurrences of $\sharp$. Therefore $w^{\prime}$ is primitive.

As $w^{\prime}$ has only one occurrence of $\sharp$, then in comparing two different cyclic shifts we necessarily compare $\sharp$ with some other character. This means that the lexicographic order of two cyclic shifts is decided no later than at the position of the first $\sharp$. Therefore if we leave out the characters after the $\sharp$ in both shifts, we get the same ordering.

Now, we give two theorems characterizing the permutations that are suffix arrays for some word $w \sharp$ with $w \in \Sigma^{n}$.

Theorem 2. Let $r_{i} \geq 0,1 \leq i \leq k$, be integers such that $\sum_{i=1}^{k} r_{i}=n$. A permutation $\pi \in \mathbf{S}_{n+1}$ is the suffix array of a word $w \sharp$ with $w \in \Sigma^{n}$ with $r_{i}$ occurrences of the letter $a_{i}, 1 \leq i \leq k$, if and only if $\mathcal{D}(\Phi(\pi)) \subseteq\left\{1,1+r_{1}, 1+r_{1}+r_{2}, \ldots, 1+r_{1}+\cdots+r_{k-1}\right\}$ and $\pi(1)=n+1$. Moreover, in this case, $\pi$ is the suffix array of exactly one such word.

Proof. According to Theorem 1 $\pi \in \mathbf{S}_{n+1}$ is the BW-array of a primitive word $w \in$ $(\Sigma \cup \sharp)^{n+1}$ which has $r_{i}$ occurrences of letter $a_{i}$ and one occurrence of symbol $\sharp$, if and only if the first condition is satisfied, and in this case there is only one such primitive word. Here the primitivity is immediate, since we have only one occurrence of $\sharp$. Since $\sharp$ is the smallest letter, condition $\pi(1)=n+1$ is necessary and sufficient for $\sharp$ to be the last letter. Finally the BW-array coincides with the suffix array on the class of words type $w \sharp$, by Proposition 3 .

From Theorem 2 we can easily deduce the following theorem:

Theorem 3. A permutation $\pi \in \mathbf{S}_{n+1}$ is the suffix array of a word $w \sharp$ where $w \in \Sigma^{n}$ if and only if (i) $|\mathcal{D}(\Phi(\pi)) \backslash\{1\}| \leq k-1$, and (ii) $\pi(1)=n+1$.

Proof. Let $\pi \in \mathbf{S}_{n+1}$ be the suffix array of a word $w \sharp$ for $w \in \Sigma^{n}$. Assume $w$ has $r_{i} \geq 0$ occurrences of letter $a_{i}$ for each $i \in[1, k]$. Then conditions $(i)$ and (ii) follow immediately from Theorem 2. Conversely, let $\mathcal{D}(\Phi(\pi)) \backslash\{1\}=\left\{d_{1}, d_{2}, \ldots, d_{\ell}\right\}$ for $\ell \leq k-1$. Then 
for $r_{1}=d_{1}-1, r_{2}=d_{2}-d_{1}, \ldots, r_{\ell}=d_{\ell}-d_{\ell-1}, r_{\ell+1}=\ldots=r_{k-1}=0$, we have $\mathcal{D}(\Phi(\pi)) \subseteq\left\{1,1+r_{1}, 1+r_{1}+r_{2}, \ldots, 1+r_{1}+\cdots+r_{k-1}\right\} . \pi(1)=n+1$ is also satisfied. Then, by Theorem 2 , there is a word $w \sharp$ with the corresponding numbers of letter occurrences that has $\pi$ as its suffix array.

Now we provide a characterization of suffix arrays for the case where we do not assume a sentinel symbol at the end of the word. Proposition 2 combined with Theorem 2 and Theorem 3 respectively imply the following results.

Theorem 4. Let $r_{i} \geq 0,1 \leq i \leq k$, be integers such that $\sum_{i=1}^{k} r_{i}=n$. A permutation $\pi \in \mathbf{S}_{n}$ is the suffix array of a word $w \in \Sigma^{n}$ with $r_{i}$ occurrences of the letter $a_{i}, 1 \leq i \leq k$, if and only if, for $\pi^{\prime}=(n+1) \pi(1) \ldots \pi(n), \mathcal{D}\left(\Phi\left(\pi^{\prime}\right)\right) \subseteq\left\{1,1+r_{1}, 1+r_{1}+r_{2}, \ldots, 1+\right.$ $\left.r_{1}+\cdots+r_{k-1}\right\}$. Moreover, in this case $\pi$ is the suffix array of exactly one such word.

Theorem 5. A permutation $\pi \in \mathbf{S}_{n}$ is the suffix array of some word $w \in \Sigma^{n}$ if and only if, for $\pi^{\prime}=(n+1) \pi(1) \ldots \pi(n)$, we have $\left|\mathcal{D}\left(\Phi\left(\pi^{\prime}\right)\right) \backslash\{1\}\right| \leq k-1$.

Finally we give a result stating a bijection between the suffix arrays over an alphabet $\Sigma$ and a certain set of permutations.

Theorem 6. For a permutation $\pi \in \mathbf{S}_{n}$, let $\pi^{\prime}=(n+1) \pi(1) \ldots \pi(n)$. The mapping $\pi \mapsto \Phi\left(\pi^{\prime}\right)$ is a bijection between the suffix arrays of words $w \in \Sigma^{n}$ and the permutations $\varphi \in \mathbf{S}_{n+1}^{c}$ with $|\mathcal{D}(\varphi) \backslash\{1\}| \leq k-1$. Moreover, given such a $\varphi \in \mathbf{S}_{n+1}^{c}$, we can easily compute the corresponding suffix array $\pi$ as follows: $\pi^{-1}(i)=\varphi^{i}(1)-1$ for each $i \in[1, n]$.

Proof. Let us denote the $\sim$ equivalence class of a $\sigma \in \mathbf{S}_{n+1}$ by $[\sigma]$. The mapping $f$ : $\mathbf{S}_{n} \rightarrow \mathbf{S}_{n+1} / \sim$ defined by $f(\pi)=\left[\pi^{\prime}\right]$ is a bijection between $\mathbf{S}_{n}$ and $\mathbf{S}_{n+1} / \sim$. $\Phi$ is bijective from $\mathbf{S}_{n+1} / \sim$ to $\mathbf{S}_{n+1}^{c}$, and hence $\pi \mapsto \Phi\left(\pi^{\prime}\right)$ is a bijection from $\mathbf{S}_{n}$ to $\mathbf{S}_{n+1}^{c}$. According to Theorem [5 a permutation $\pi \in \mathbf{S}_{n}$ is a suffix array of some word in $\Sigma^{n}$ if and only if, for its its image $\Phi\left(\pi^{\prime}\right),\left|\mathcal{D}\left(\Phi\left(\pi^{\prime}\right)\right) \backslash\{1\}\right| \leq k-1$. Then it follows that the restriction of the mapping to the set of suffix permutations gives a bijection into the set of permutations $\varphi \in \mathbf{S}_{n+1}^{c}$ with $|\mathcal{D}(\varphi) \backslash\{1\}| \leq k-1$.

As for the computation of the inverse mapping, we know that $\left(\pi^{\prime}\right)^{-1}(n+1)=1$ and that $\Phi\left(\pi^{\prime}\right)\left(\left(\pi^{\prime}\right)^{-1}(i)\right)=\left(\pi^{\prime}\right)^{-1}(i+1)$. Therefore, if $\varphi=\Phi\left(\pi^{\prime}\right)$, then $\pi^{-1}(i)=$ $\left(\pi^{\prime}\right)^{-1}(i)-1=\varphi^{i}(1)-1$ for all $i \in[1, n]$.

\section{Relation to the characterization of $\mathrm{He}$ et al}

He et al. 2] proposed a characterization of suffix arrays for a binary alphabet $\Sigma=$ $\{a, b\}$ in the special case where the sentinel character $\sharp$ is ranked between the characters of $\Sigma$, i.e., $a<\sharp<b$. In this case, the lexicographic order of suffixes of $w$ can be different from the lexicographic order of the corresponding suffixes of $w \sharp$, therefore this definition gives a slightly different suffix array notion.

In this section, we elucidate how the characterization of [2] is related to our characterizations given in Section 3. In particular, we show that our approach yields a simpler characterization that implies the result of [2]. Before describing the characterization of [2], we show that Theorem 1 allows us to obtain a characterization of suffix arrays for this kind of alphabet ordering as well, similarly to the usual ordering of the previous section. 
Theorem 7. A permutation $\pi \in \mathbf{S}_{n+1}$ is the suffix array of a word $w \sharp$ with $w \in\{a, b\}^{n}$ and $a<\sharp<b$ if and only if $\mathcal{D}(\Phi(\pi)) \subseteq\left\{\pi^{-1}(n+1)-1, \pi^{-1}(n+1)\right\}$.

Proof. Let $\pi \in \mathbf{S}_{n+1}$. By Proposition 3 , $\pi$ is the suffix array of $w \sharp$ if and only if it is the $\mathrm{BW}$-arrays of $w \sharp$. Therefore it is enough to prove the theorem for BW arrays instead of suffix arrays.

We first show the 'only if' part. Observe that if $\pi$ is the BW array of $w \sharp$, then $w_{\pi(i)}=a$ for $i<\pi^{-1}(n+1)$, and $w_{\pi(i)}=b$ for $i>\pi^{-1}(n+1)$. Therefore $w$ has $\pi^{-1}(n+1)-1$ occurrences of $a, 1$ occurrence of $\sharp$ and $n+1-\pi^{-1}(n+1)$ occurrences of b. By Theorem 1, we immediately obtain $\mathcal{D}(\Phi(\pi)) \subseteq\left\{\pi^{-1}(n+1)-1, \pi^{-1}(n+1)\right\}$.

We now prove the 'if' part. Suppose that $\mathcal{D}(\Phi(\pi)) \subseteq\left\{\pi^{-1}(n+1)-1, \pi^{-1}(n+1)\right\}$. From Theorem 1 there exists exactly one word $w^{\prime} \in\{a, \sharp, b\}^{n+1}$ that has $\pi^{-1}(n+1)-1$ occurrences of $a, 1$ occurrence of $\sharp$ and $n+1-\pi^{-1}(n+1)$ occurrences of $b$ and which has $\pi$ as BW array. From $w_{\pi(1)}^{\prime} \leq w_{\pi(2)}^{\prime} \leq \cdots \leq w_{\pi(n+1)}^{\prime}$, this word is the following: $w_{\pi(i)}^{\prime}=a$ for $i<\pi^{-1}(n+1), w_{\pi\left(\pi^{-1}(n+1)\right)}^{\prime}=w_{n+1}^{\prime}=\sharp$, and $w_{\pi(i)}^{\prime}=b$ for $i>\pi^{-1}(n+1)$. We can see, that $w^{\prime}=w \sharp$ where $w \in\{a, b\}^{n}$, therefore we have the sufficiency of the condition.

Now, we repeat the characterization given by He et al. [2]. We need some additional definitions.

Definition 7 (Ascending-to-max [2]). A permutation $\pi \in \mathbf{S}_{n+1}$ is ascending-to-max if and only if, for every $i \in[1, n-1]$, we have

(a) if $\pi^{-1}(i)<\pi^{-1}(n+1), \pi^{-1}(i+1)<\pi^{-1}(n+1)$, then $\pi^{-1}(i)<\pi^{-1}(i+1)$, and

(b) if $\pi^{-1}(i)>\pi^{-1}(n+1), \pi^{-1}(i+1)>\pi^{-1}(n+1)$, then $\pi^{-1}(i)>\pi^{-1}(i+1)$.

Definition 8 (Non-nesting [2]). A permutation $\pi \in \mathbf{S}_{n+1}$ is non-nesting if and only if, for each $i, j \in[1, n]$ such that $\pi^{-1}(i)<\pi^{-1}(j)$, if

(a) $\pi^{-1}(i)<\pi^{-1}(i+1)$ and $\pi^{-1}(j)<\pi^{-1}(j+1)$, or

(b) $\pi^{-1}(i)>\pi^{-1}(i+1)$ and $\pi^{-1}(j)>\pi^{-1}(j+1)$,

then $\pi^{-1}(i+1)<\pi^{-1}(j+1)$.

The characterization of [2] is as follows.

Theorem 8 ([2]). A permutation $\pi \in \mathbf{S}_{n+1}$ is the suffix array of a word $w \sharp$ with $w \in$ $\{a, b\}^{n}$ and $a<\sharp<b$ if and only if it is both ascending-to-max and non-nesting.

We now show that the condition of Theorem 8 is equivalent to that of Theorem 7 . Let $\varphi=\Phi(\pi)$. We have $\varphi\left(\pi^{-1}(i)\right)=\pi^{-1}(i+1)$. Therefore, the ascending-to-max property reduces to $i<\varphi(i)$ for $i \in\left[1, \pi^{-1}(n+1)-1\right]$, and $i>\varphi(i)$ for $i \in\left[\pi^{-1}(n+1)+1, n+1\right]$. As for the non-nesting property, we have the following: for $i, j \in[1, n] \backslash\left\{\pi^{-1}(n+1)\right\}$, if $i<\varphi(i)$ and $j<\varphi(j)$, or $i>\varphi(i)$ and $j>\varphi(j)$, then $i<j$ implies $\varphi(i)<\varphi(j)$.

We show that the two conditions together are equivalent to the condition of Theorem 7 . The two conditions together trivially imply the condition of Theorem 7 . Conversely, suppose that $\mathcal{D}(\Phi(\pi)) \subseteq\left\{\pi^{-1}(n+1)-1, \pi^{-1}(n+1)\right\} . \varphi$ has one orbit, and hence $\varphi(1)>1$. If for some $j \in[1, n]$ we have $\varphi(j)>j$ and $\varphi(j+1)<j+1$, then $j$ is a 
descent. Hence for $i \in\left[1, \pi^{-1}(n+1)-1\right], i<\varphi(i)$. Similarly,for $i \in\left[\pi^{-1}(n+1)+1, n+1\right]$, $i>\varphi(i)$. From $\mathcal{D}(\Phi(\pi)) \subseteq\left\{\pi^{-1}(n+1)-1, \pi^{-1}(n+1)\right\}$ it follows that $\varphi$ is monotone on $\left[1, \pi^{-1}(n+1)-1\right]$ and on $\left[\pi^{-1}(n+1)+1, n+1\right]$, and hence the non-nesting property is also satisfied.

\section{Enumerations}

Our characterization theorems from Section 3 can also be used to count objects related to suffix arrays. Schürmann and Stoye [3] obtained some counting results using "direct" combinatorial considerations. Here we give shorter proofs of these results, based on bijections provided by Theorems 446 from Section 3 , The underlying ideas of the proofs are the same as in [3], the simplification is due to the more simple characterization of suffix arrays.

The following enumerations have been studied in [3].

(i) For a permutation $\pi \in \mathbf{S}_{n}$, count the number of words of length $n$ over an alphabet of size $k$ that have $\pi$ as their suffix array,

(ii) For a permutation $\pi \in \mathbf{S}_{n}$, count the number of words of length $n$ over an alphabet of size $k$, that have at least one occurrence of each letter and have $\pi$ as their suffix array,

(iii) Count the number of permutations $\pi \in \mathbf{S}_{n}$ that are suffix arrays of some word over an alphabet of size $k$.

We start with question (i).

Theorem 9 (3]). For a permutation $\pi \in \mathbf{S}_{n}$, let $\pi^{\prime}=(n+1) \pi(1) \ldots \pi(n)$. The number of words of length $n$ over an alphabet of size $k$ having $\pi$ as their suffix array is

$$
\left(\begin{array}{c}
n+k-1-\left|\mathcal{D}\left(\Phi\left(\pi^{\prime}\right)\right) \backslash\{1\}\right| \\
k-1-\left|\mathcal{D}\left(\Phi\left(\pi^{\prime}\right)\right) \backslash\{1\}\right|
\end{array}\right) .
$$

Proof. Theorem 4 states that if $r_{i} \geq 0$ for $i=1 \ldots k, \sum_{i=1}^{k} r_{i}=n$, and

$$
\mathcal{D}\left(\Phi\left(\pi^{\prime}\right)\right) \subseteq\left\{1,1+r_{1}, 1+r_{1}+r_{2}, \ldots, 1+r_{1}+\cdots+r_{k-1}\right\},
$$

then there is exactly one word $w$ with $r_{i}$ occurrences of $a_{i}$ that has $\pi$ as its suffix array. Therefore, we need to count the number of tuples $\left(r_{1}, \ldots, r_{k}\right)$ (Parikh vectors) that satisfy (1) given a permutation $\pi^{\prime}=(n+1) \pi(1) \ldots \pi(n)$. We represent a tuple $\left(r_{1}, \ldots, r_{k}\right), \sum_{i=1}^{k} r_{i}=n$, as a sequence of $n$ dots divided into $k$ (possibly empty) groups separated by $k-1$ separators:

$$
\left(r_{1}, \ldots, r_{k}\right), \sum r_{i}=n \quad \leftrightarrow \quad \underbrace{0 \cdots 0}_{r_{1}}|\underbrace{0 \cdots 0}_{r_{2}}| \cdots \mid \underbrace{0 \cdots 0}_{r_{k}}
$$

Clearly, this representation is a bijection.

If $i>1$ is a descent of $\Phi\left(\pi^{\prime}\right)$, there must be a separator between the $(i-1)$-th and the $i$-th dots. This defines the placement of $\left|\mathcal{D}\left(\Phi\left(\pi^{\prime}\right)\right) \backslash\{1\}\right|$ separators. The remaining 
$\left(k-1-\left|\mathcal{D}\left(\Phi\left(\pi^{\prime}\right)\right) \backslash\{1\}\right|\right)$ separators can interleave the $n$ dots arbitrarily. This can be done in

$$
\left(\begin{array}{c}
n+k-1-\left|\mathcal{D}\left(\Phi\left(\pi^{\prime}\right)\right) \backslash\{1\}\right| \\
k-1-\left|\mathcal{D}\left(\Phi\left(\pi^{\prime}\right)\right) \backslash\{1\}\right|
\end{array}\right)=\left(\begin{array}{c}
n+k-1-\left|\mathcal{D}\left(\Phi\left(\pi^{\prime}\right)\right) \backslash\{1\}\right| \\
n
\end{array}\right)
$$

ways. It is easy to get convinced that the construction provides a bijection with the considered set of tuples. The result follows.

Note that if $k-1<\left|\mathcal{D}\left(\Phi\left(\pi^{\prime}\right)\right) \backslash\{1\}\right|$, there is no word on an alphabet of size $k$ which has $\pi$ as its suffix array. This is confirmed by Proposition 9, as $\left(\begin{array}{c}m \\ n\end{array}\right)=0$ for $m<n$. The following proposition from [3] answers question (ii).

Theorem $10([3])$. For a permutation $\pi \in \mathbf{S}_{n}$, let $\pi^{\prime}=(n+1) \pi(1) \ldots \pi(n)$. The number of words of length $n$ over an alphabet of size $k$ that have at least one occurrence of each of the $k$ letters and have $\pi$ as their suffix array is

$$
\left(\begin{array}{l}
n-1-\left|\mathcal{D}\left(\Phi\left(\pi^{\prime}\right)\right) \backslash\{1\}\right| \\
k-1-\left|\mathcal{D}\left(\Phi\left(\pi^{\prime}\right)\right) \backslash\{1\}\right|
\end{array}\right)
$$

Proof. We modify the proof of Proposition 9 to insure that that each letter occurs at least once. We cannot have two adjacent separators, and we cannot start or end with a separator. We then have to distribute $k-1$ separators among $n-1$ possible places between the circles. Like in the proof of Proposition 9, the place of $\left|\mathcal{D}\left(\Phi\left(\pi^{\prime}\right)\right) \backslash\{1\}\right|$ separators is determined by $\pi^{\prime}$, and the remaining $\left(k-1-\left|\mathcal{D}\left(\Phi\left(\pi^{\prime}\right)\right) \backslash\{1\}\right|\right)$ separators are distributed among $\left(n-1-\left|\mathcal{D}\left(\Phi\left(\pi^{\prime}\right)\right) \backslash\{1\}\right|\right)$ remaining places. This yields the count of the Theorem.

Finally, we give a proof for question (iii), based on the results of Section 3 , Let $\left\langle\begin{array}{l}n \\ d\end{array}\right\rangle$ denote the Eulerian number, i.e. the number of permutations of $[1, n]$ with exactly $d$ descents.

Theorem 11 ([3]). The number of permutations $\pi \in \mathbf{S}_{n}$ that are suffix arrays of a word $w \in \Sigma^{n}$ with $|\Sigma|=k$ is $\sum_{d=0}^{k-1}\left\langle\begin{array}{l}n \\ d\end{array}\right\rangle$.

Proof. According to Theorem [6, there is a bijection between the suffix arrays of words $w \in \Sigma^{n}$ and the permutations $\varphi \in \mathbf{S}_{n+1}^{c}$ such that $|\mathcal{D}(\varphi) \backslash\{1\}| \leq k-1$. We then have to count the number of such permutations. Let $P(n, d)$ denote the number of permutations $\varphi \in \mathbf{S}_{n+1}^{c}$ with $|\mathcal{D}(\varphi) \backslash\{1\}|=d$. To prove the theorem, we show that $P(n, d)$ is equal to the Eulerian number $\left\langle\begin{array}{l}n \\ d\end{array}\right\rangle$.

The proof is by induction on $n$. Trivially, $P(1,0)=1=\left\langle\begin{array}{l}1 \\ 0\end{array}\right\rangle$ (the only good permutation is $\pi=21)$, and $P(1, d)=0=\left\langle\begin{array}{l}1 \\ d\end{array}\right\rangle$ when $d \geq 1$. We now show that $P(n, d)=(d+1) P(n-$ $1, d)+(n-d) P(n-1, d-1)$, thereby proving that $P(n, d)=\left\langle\begin{array}{l}n \\ d\end{array}\right\rangle$. For the inductive step, 


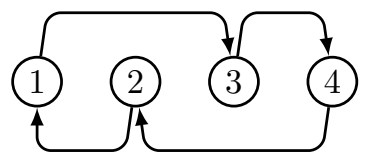

$\varphi$

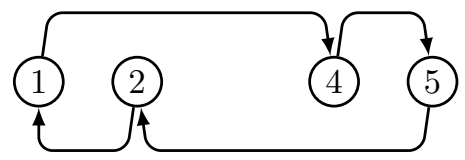

$\operatorname{Aug}_{s} \circ \varphi \circ \operatorname{Aug}_{s}^{-1}$

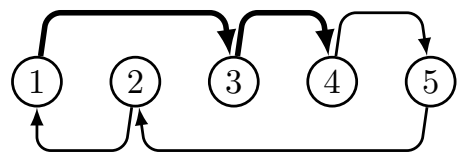

$T_{s}(\varphi)$

Figure 1: Illustration of $T_{s}(\varphi)$ with $\varphi=3142$ and $s=3$. Informally, $\operatorname{Aug}_{s} \circ \varphi \circ \operatorname{Aug}_{s}^{-1}$ "increments by one" all nodes $s, \ldots, n$. Then $T_{s}(\varphi)$ "splits" the mapping $(1, \varphi(1))$ into $(1, s)$ and $(s, \varphi(1))$.

we describe a generative procedure for the considered permutations. Consider $\varphi \in \mathbf{S}_{n}^{c}$ and let $s \in[2, n+1]$. Consider the mapping $\operatorname{Aug}_{s}:[1, n] \rightarrow[1, n+1]$ defined by

$$
\operatorname{Aug}_{s}(i)= \begin{cases}i & \text { if } i<s \\ i+1 & \text { if } i \geq s\end{cases}
$$

Observe that $\operatorname{Aug}_{s} \circ \varphi \circ \operatorname{Aug}_{s}^{-1}$ is bijective on the set $\{1, \ldots, s-1, s+1, \ldots, n\}$ and has one orbit. Now consider the transform $T_{s}: \mathbf{S}_{n}^{c} \rightarrow \mathbf{S}_{n+1}^{c}$ defined by

$$
T_{s}(\varphi)(i)= \begin{cases}\operatorname{Aug}_{s} \circ \varphi \circ \operatorname{Aug}_{s}^{-1}(i) & \text { if } i \in[1, n+1] \backslash\{1, s\}, \\ s & \text { if } i=1, \\ \operatorname{Aug}_{s} \circ \varphi \circ \operatorname{Aug}_{s}^{-1}(1)=\varphi(1) & \text { if } i=s .\end{cases}
$$

It is straightforward to check that $T_{s}(\varphi) \in \mathbf{S}_{n+1}^{c}$, i.e., $T_{s}(\varphi) \in \mathbf{S}_{n+1}$ and it has one orbit. The construction is illustrated in Figure 1.

Furthermore, if $r, s \in[2, n+1]$ and $\varphi, \psi \in \mathbf{S}_{n}^{c}$, where $r \neq s$ or $\varphi \neq \psi$, then $T_{s}(\varphi) \neq$ $T_{r}(\psi)$. As there are $(n-1)$ ! permutations in $\mathbf{S}_{n}^{c}$, we get $n \cdot(n-1) !=n$ ! different permutations $T_{s}(\varphi)$ for $s \in[2, n+1]$ and $\varphi \in \mathbf{S}_{n}^{c}$. Therefore, $\mathbf{S}_{n+1}^{c}=\left\{T_{s}(\varphi): s \in\right.$ $\left.[2, n+1], \varphi \in \mathbf{S}_{n}^{c}\right\}$.

Now, we examine how the transform $T_{s}(\varphi)$ affects the number of descents of $\varphi$. For $i \in[2, n-1] \backslash\{s-1\}, T_{s}(\varphi)\left(\operatorname{Aug}_{s}(i)\right)=\operatorname{Aug}_{s}(\varphi(i))$ and $T_{s}(\varphi)\left(\operatorname{Aug}_{s}(i)+1\right)=$ $T_{s}(\varphi)\left(\operatorname{Aug}_{s}(i+1)\right)=\operatorname{Aug}_{s}(\varphi(i+1))$. Therefore for $i \in[2, n-1] \backslash\{s-1\}$

$$
\operatorname{Aug}_{s}(i) \in \mathcal{D}\left(T_{s}(\varphi)\right) \Leftrightarrow i \in \mathcal{D}\left(\operatorname{Aug}_{s}(\varphi)\right) \Leftrightarrow i \in \mathcal{D}(\varphi),
$$

where the second equivalence follows from the monotonicity of $\mathrm{Aug}_{s}$. Thus, $\mathrm{Aug}_{s}$ gives a one-to-one correspondence between $\mathcal{D}(\varphi) \backslash\{1, s-1\}$ and $\left.\mathcal{D}\left(T_{s}(\varphi)\right) \backslash\{1, s-1, s\}\right)$. It remains to analyze values $s-1$ and $s$. We have $T_{s}(\varphi)(s+1)=\operatorname{Aug}_{s}(\varphi)(s)<\operatorname{Aug}_{s}(\varphi)(s-$ $1)=T_{s}(\varphi)(s-1)$ if and only if $s-1 \in \mathcal{D}(\varphi)$. In this case, $s-1$ or $s$ is a descent of $T_{s}(\varphi)$. The insertion of $T_{s}(\varphi)(s)=\varphi(1)$ may or may not create a new descent. For a given $\varphi \in \mathbf{S}_{n}^{c}$, in each monotonic run of $\varphi$ on indices $\{2, \ldots, n\}$, there is exactly one position where we can place $\varphi(1)$ without creating a new descent, otherwise we create exactly one new descent.

How many $T_{s}(\varphi)$ can we have with $\left|\mathcal{D}\left(T_{s}(\varphi)\right) \backslash\{1\}\right|=d$ ? For each $\varphi \in \mathbf{S}_{n}^{c}$ with $|\mathcal{D}(\varphi) \backslash\{1\}|=d$, we have $(d+1)$ possibilities to choose $s$ ( $\varphi$ has $d+1$ monotonic runs on $\{2, \ldots, n\})$. For each $\varphi \in \mathbf{S}_{n}^{c}$ with $|\mathcal{D}(\varphi) \backslash\{1\}|=d-1$, we have $(n-d)$ possibilities to choose $s$. These permutations are all different as $T_{s}(\varphi) \neq T_{r}(\psi)$ if $s \neq r$ or $\varphi \neq \psi$. There 
is no other way to get a permutation $\psi \in \mathbf{S}_{n+1}^{c}$ with $|\mathcal{D}(\psi) \backslash\{1\}|=d$. We conclude that $P(n, d)=(d+1) P(n-1, d)+(n-d) P(n-1, d-1)$. This proves the Theorem.

\section{References}

[1] M. Crochemore, J. Désarménien, D. Perrin, A note on the Burrows-Wheeler transformation, Theor. Comput. Sci. 332 (1-3) (2005) 567-572.

[2] M. He, J. I. Munro, S. S. Rao, A categorization theorem on suffix arrays with applications to space efficient text indexes, in: Proceedings of the sixteenth annual ACM-SIAM symposium on Discrete algorithms, SODA'05, Society for Industrial and Applied Mathematics, Philadelphia, PA, USA, 23-32, 2005.

[3] K.-B. Schürmann, J. Stoye, Counting suffix arrays and strings, Theoretical Computer Science 395 (2008) $220-234$.

[4] U. Manber, E. W. Myers, Suffix Arrays: A New Method for On-Line String Searches, SIAM J. Comput. 22 (5) (1993) 935-948.

[5] G. H. Gonnet, R. A. Baeza-Yates, T. Snider, New Indices for Text: Pat Trees and Pat Arrays, in: Information Retrieval: Data Structures \& Algorithms, Prentice-Hall, 66-82, 1992.

[6] J. Kärkkäinen, P. Sanders, Simple Linear Work Suffix Array Construction, in: J. C. M. Baeten, J. K. Lenstra, J. Parrow, G. J. Woeginger (Eds.), Automata, Languages and Programming, Proc. of the 30th International Colloquium, ICALP 2003, Eindhoven, The Netherlands, June 30 - July 4, 2003., vol. 2719 of Lecture Notes in Computer Science, Springer, 943-955, 2003.

[7] D. K. Kim, J. S. Sim, H. Park, K. Park, Linear-Time Construction of Suffix Arrays, in: R. A. Baeza-Yates, E. Chávez, M. Crochemore (Eds.), Combinatorial Pattern Matching, Proc. of the 14th Annual Symposium, CPM 2003, Morelia, Michocán, Mexico, June 25-27, 2003., vol. 2676 of Lecture Notes in Computer Science, Springer, 186-199, 2003.

[8] P. Ko, S. Aluru, Space Efficient Linear Time Construction of Suffix Arrays, in: R. A. Baeza-Yates, E. Chávez, M. Crochemore (Eds.), Combinatorial Pattern Matching, Proc. of the 14th Annual Symposium, CPM 2003, Morelia, Michocán, Mexico, June 25-27, 2003., vol. 2676 of Lecture Notes in Computer Science, Springer, 200-210, 2003.

[9] M. Burrows, D. Wheeler, A block sorting lossless data compression algorithm, Tech. Rep., Systems Research Center, Technical Report 124, Digital Equipment Corporation, 1994.

[10] G. Navarro, V. Mäkinen, Compressed full-text indexes, ACM Comput. Surv. 39 (1).

[11] I. M. Gessel, C. Reutenauer, Counting Permutations with Given Cycle Structure and Descent Set, J. Comb. Theory, Ser. A 64 (2) (1993) 189-215.

[12] R. Grossi, J. S. Vitter, Compressed Suffix Arrays and Suffix Trees with Applications to Text Indexing and String Matching, SIAM J. Comput. 35 (2) (2005) 378-407.

[13] H. Bannai, S. Inenaga, A. Shinohara, M. Takeda, Inferring Strings from Graphs and Arrays, in: B. Rovan, P. Vojtás (Eds.), Mathematical Foundations of Computer Science 2003, Proc. of the 28th International Symposium, MFCS 2003, Bratislava, Slovakia, August 25-29, 2003., vol. 2747 of Lecture Notes in Computer Science, Springer, 208-217, 2003. 